\title{
Especies con potencial para sistemas agroforestales en el Departamento del Magdalena, Colombia
}

\author{
Sonia E. Aguirre-Forero, Nelson V. Piraneque-Gambasica* y Cristian F. Abaunza-Suárez \\ Universidad del Magdalena, Santa Marta - Colombia (correo-e: saguirre@unimagdalena.edu.co; \\ npiraneque@unimagdalena.edu.co; cristianabaunzafs@unimagdalena.edu.co)
}

${ }^{*}$ Autor a quien debe ser dirigida la correspondencia

Recibido Feb. 3, 2021; Aceptado Abr. 1, 2021; Versión final May. 13, 2021, Publicado Ago. 2021

\section{Resumen}

El objetivo principal de esta investigación fue identificar especies con potencial para incorporarse a sistemas agroforestales en 63 unidades productivas de ocho municipios del departamento del Magdalena (Colombia). Mediante recorridos en campo y el apoyo de los productores, se realizó el reconocimiento de la composición de especies vegetales presentes en los sistemas productivos, seguido de su identificación empleando claves taxonómicas y así, obtener el inventario de las mismas, el hábito de crecimiento y los usos más comunes. Los resultados reportaron 122 especies, de las cuales el $66 \%$ son árboles, el $28 \%$ hierbas terrestres y el $6 \%$ arbustos. El $81 \%$ de las especies sirven para sombra, cercas vivas, forrajes, medicinales, ornamentales y protectoras de fuentes de agua, mientras que $35 \%$ son maderables y $29 \%$ forrajeras. Se concluye que las especies seleccionadas presentan características deseables para sistemas agroforestales en el trópico seco.

Palabras clave: sector agropecuario; sistemas agroforestales; árboles; arbustos; especies

\section{Species with agroforestry-systems potential in the Magdalena state, Colombia}

\begin{abstract}
The primary objective of this research study was to identify plant species with the potential to be incorporated into agroforestry systems. The present study was conducted in 63 productive units located in eight municipalities in the Magdalena state (Colombia). The plant species composition present in the agricultural systems was characterized in field trips supported by local farmers. Plant taxonomic keys were used to identify and create an inventory that included information on growth, habits, and most common uses. One hundred and twenty-two species were identified: $66 \%$ were trees, $28 \%$ were terrestrial herbs, and $6 \%$ were shrubs. Eighty-one percent of the species were used as shade, living fences, forage, medicine, ornaments, and for preservation of water sources, while $35 \%$ were timber and $29 \%$ were forage. It was concluded that the species selected have desirable traits for agroforestry systems in the dry tropics.
\end{abstract}

Keywords: agricultural sector; agroforestry systems; trees; shrubs; species 


\section{INTRODUCCIÓN}

La ganadería, es uno de los tensores de cambio climático toda vez que está relacionada con deforestación, deterioro de los recursos suelo, agua y biodiversidad y, por tanto, de la reducción en los servicios ecosistémicos (Mancera et al., 2018). Debido a esto, en el mundo se pierden más de 129 millones de hectáreas forestales que, unido al crecimiento poblacional y la necesidad de mayor producción de alimentos, se agudiza por el inadecuado manejo de los ecosistemas para la explotación ganadera y de monocultivos, con prácticas irracionales que producen deterioro ambiental y disminuyen la producción agropecuaria (Revueltas et al., 2020), comprometiendo la seguridad alimentaria y el bienestar de generaciones venideras (Buitrago-Guillen et al., 2018).

En Colombia existen más de 34 millones de ha dedicadas a la actividad ganadera $(85 \%$ en ganadería extensiva) y de éstas, 21 millones de hectáreas tienen potencialidad para usos agroforestales esto es, el 19,3 $\%$ del territorio nacional (Mazo et al., 2016). Infortunadamente, la tala y quemas, generan fragmentación y perdida de numerosas especies nativas, con aumento progresivo de gases efecto invernadero - GEI tales como $\mathrm{CO}_{2}, \mathrm{NO}_{2}$ y metano (Buitrago-Guillen et al., 2018) y se promueve el cambio de uso de suelo y la degradación de la biodiversidad (Díaz-Céspedes et al., 2020).

En la región caribe colombiana, la actividad pecuaria es ineficiente, traducido en baja rentabilidad del negocio y el impacto de la actividad, por lo que es necesario cambiar hábitos y mejorar la tecnología de producción pecuaria (Mazo et al., 2016). Para esto, se debe identificar especies perennes productoras de biomasa que diversifiquen e imiten la eficiencia del bosque natural y ofrezcan aporte nutricional a los animales, además de sombrío y ciclaje de materia orgánica que contribuya a mantener suelos cubiertos con especies fijadoras de nitrógeno y carbono (poáceas y otras familias), protectoras de cuencas hidrográficas, generadoras de alimento y hábitat de diversas especies, como son algunos árboles y arbustos con múltiples usos que se adaptan y viven en la zona (Mancera et al., 2018). Los forrajes mejorados como las poáceas, contribuyen al aumento de rendimientos del sector, no obstante, su manejo como pasto es dispendioso y de alto costo (Cardona et al., 2012) y las condiciones climáticas extremas de calor del trópico seco, las afecta significativamente.

Las características edafo-climáticas y las prácticas de manejo de las actividades agropecuarias en el departamento del Magdalena, aumentan el grado de vulnerabilidad del territorio al fenómeno de cambio climático, con consecuencias como la escasez de forrajes (Revueltas et al., 2020). El déficit de lluvias, alta temperatura y la sequía, reducen la producción y provoca muerte de ganado. Ante esta situación, es necesario implementar sistemas mixtos integrados de árboles, arbustos forrajeros o multipropósito para corte y fomentar ciclaje de biomasa en praderas con sombrío, como alternativas viables y sostenibles (Mancera et al., 2018; Mazo et al., 2016). De acuerdo con lo mencionado, los sistemas agroforestales, son una alternativa de producción ganadera en la costa Caribe colombiana (Revueltas et al., 2020) al restablecer la cobertura vegetal y generar crecimiento espontáneo de herbáceas, árboles y arbustos adaptados a estos ecosistemas (Londoño y Torres, 2015).

Los sistemas agroforestales, permiten aprovechar las ventajas de varios estratos de la vegetación (sistemas donde existe combinación de especies arbóreas y arbustivas o herbáceas) y aumenta la biodiversidad (Buitrago-Guillen et al., 2018), siendo el silvopastoreo, una modalidad de producción pecuaria donde plantas leñosas perennes (árboles y/o arbustos) interactúan con componentes tradicionales (forrajeras, herbáceas y animales) bajo un sistema de manejo integral, que considera la composición de diferentes sistemas de producción de ganado bovino (Mancera et al., 2018), siendo alternativa para mejorar la producción pecuaria y prevenir procesos de degradación de suelos (Mazo et al., 2016) y, ante la escaza investigación en el área, es necesario identificar de especies para incorporarlas a los sistemas ganaderos del trópico seco del departamento del Magdalena, que optimicen la ganadería en las condiciones agroclimáticas del área de estudio y favorezcan la revegetalización del territorio, como uno de los retos del desarrollo sostenible del sector (Revueltas et al., 2020).

Bajo esta perspectiva, este estudio se planteó con el objetivo de identificar, caracterizar y analizar los posibles usos de arbustos y árboles adaptados a las condiciones edáficas y climáticas del departamento del Magdalena. Información que es fundamental para priorizar modelos agroforestales, como alternativas de mitigación a fenómenos climáticos cambiantes, bajo un concepto de producción agropecuaria más sostenible.

\section{METODOLOGÍA}

La metodología se describe en tres sub-secciones para mejor presentación de las distintas etapas: i) la localización de la investigación; ii) el enfoque metodológico; y iii) el análisis estadístico realizado. 


\section{Área de estudio}

El estudio se desarrolló en 63 unidades productivas (UP) pertenecientes a cuatro zonas (Norte, Río, Centro y Sur) y ocho municipios: Zona Bananera, Chivolo, Sabanas de San Ángel, Santa Bárbara de Pinto, Plato, Nueva Granada, Guamal y Sitio Nuevo (Figura 1) del Departamento del Magdalena, localizado al norte del Colombia (08 $54^{\prime} 59^{\prime \prime}$ y $11^{\circ} 20^{\prime} 58^{\prime \prime}$ de latitud Norte, y $73^{\circ} 32^{\prime} 32^{\prime \prime}$ y $74^{\circ} 56^{\prime} 51^{\prime \prime}$ de longitud Oeste), área que presenta diversos ecosistemas que van desde bosque seco tropical (bs-T), hasta bosque húmedo tropical (bh-T) (Revueltas et al., 2020).

La parte plana del departamento presenta lluvias entre 1000 y $1500 \mathrm{~mm}$. Al extremo sur, en el sector del municipio de El Banco, y en las estribaciones de la Sierra Nevada, las lluvias se incrementan hasta valores superiores de $2000 \mathrm{~mm}$. El régimen de lluvias del departamento presenta dos temporadas, la primera en abril y mayo, la segunda en septiembre y noviembre, una temporada de menor intensidad de lluvias entre los meses de junio y agosto y, por último, una temporada seca entre los meses de diciembre a marzo. Se registran temperaturas superiores a los $28^{\circ} \mathrm{C}$ en el sur del departamento, mientras que al centro y norte los valores oscilan entre 26 y $28{ }^{\circ} \mathrm{C}$. Sobre la Sierra Nevada, la temperatura se reduce en función de la altura, descendiendo hasta menos de $8^{\circ} \mathrm{C}$, en las partes más altas (Aguirre et al., 2019; Velándia et al., 2009).

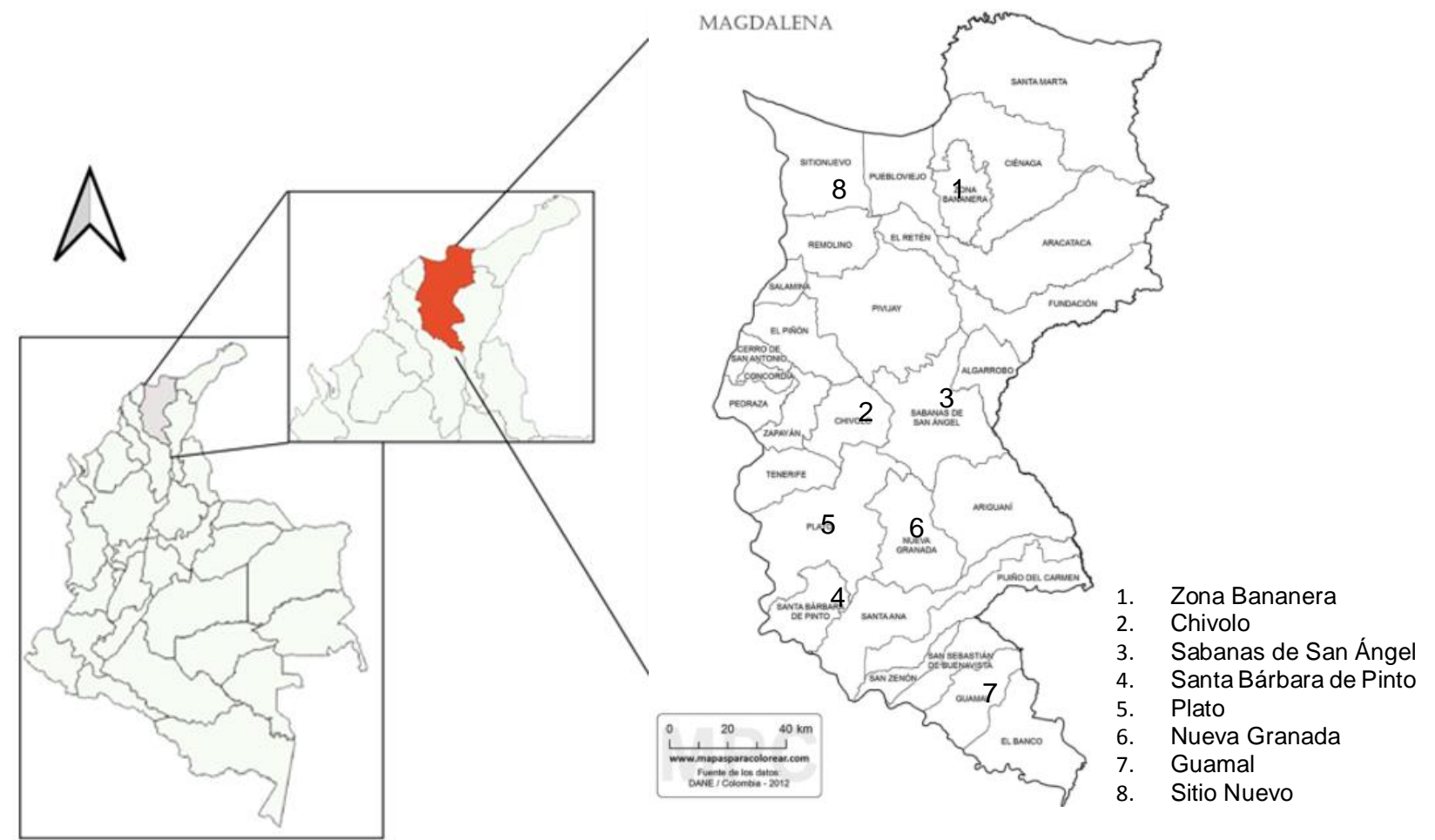

Fig. 1: Municipios de las zonas Norte, Río, Centro y Sur donde se localizó el área de estudio.

\section{Enfoque metodológico}

Mixto, investigación básica y descriptiva. La primera etapa de la investigación se realizó en campo. Mediante observación directa y apoyo de los productores de la zona, se efectuó el reconocimiento de la composición de especies vegetales presentes en los sistemas productivos de 63 UP de ocho municipios y cuatro zonas del departamento definidas por las características edafo-climáticas establecidas por Velándia et al. (2009) (Tabla 1). En la Tabla, A es la altura sobre el nivel del mar, $P$ es la precipitación media anual, ETP es la evapotranspiración potencial media anual, y T es la (temperatura media anual

\section{Muestreos}

El inventario vegetal se realizó a partir de 189 muestreos (seis por UP), siguiendo rutas en zigzag en cada finca. Cada UP fue visitada dos veces durante el año 2020 para colección de datos. En cada una, se recolectaron hojas, semillas y flores, se realizó registro fotográfico para posterior clasificación de las especies junto con información básica como localización, nombre común y los diferentes usos derivados del conocimiento local. Para cada especie se consideró una distancia de $50 \mathrm{~m}$ entre individuos a fin de evitar altos valores de parentesco de acuerdo a lo descrito por Bocanegra-González et al. (2019). 
Tabla 1: Descripción edafo-climática de las zonas en estudio. (Adaptada de Velándia et al., 2009).

\begin{tabular}{|c|c|c|c|}
\hline Zona & Municipio (s) & Clima & Suelos \\
\hline Norte & Zona Bananera & $\begin{array}{l}\text { Cálido seco, A } 20 \mathrm{~m}, \mathrm{P} 1121 \mathrm{~mm} \text {, } \\
\text { ETP } 1893 \mathrm{~mm}, \mathrm{~T} 27.6^{\circ} \mathrm{C}\end{array}$ & $\begin{array}{l}\text { Profundos, bien drenados, texturas medias } \\
\text { a moderadamente gruesas, } \\
\text { moderadamente ácidos a fuertemente } \\
\text { alcalinos, alta saturación de bases, fertilidad } \\
\text { natural alta a moderada }\end{array}$ \\
\hline Río & Sitio Nuevo & $\begin{array}{l}\text { Cálido seco, A } 5 \mathrm{~m}, \text { P } 865 \mathrm{~mm} \text {, ETP } \\
1741.2 \mathrm{~mm}, \mathrm{~T} 27^{\circ} \mathrm{C}\end{array}$ & $\begin{array}{l}\text { Superficiales y profundos, bien drenados, } \\
\text { texturas medias a finas, fuertemente ácidos } \\
\text { a ligeramente alcalinos, alta saturación de } \\
\text { bases, fertilidad natural baja a alta. }\end{array}$ \\
\hline Centro & $\begin{array}{l}\text { Plato } \\
\text { Nueva Granada } \\
\text { Chivolo } \\
\text { Sabanas de } \\
\text { San Ángel }\end{array}$ & $\begin{array}{l}\text { Cálido seco, A } 20 \mathrm{~m}, \mathrm{P} 910 \mathrm{~mm} \text {, } \\
\text { ETP } 1739 \mathrm{~mm}, \mathrm{~T}^{\circ} \mathrm{C} \text {. } \\
\text { Cálido, A } 30 \mathrm{~m}, \mathrm{P} 1400 \mathrm{~mm} \text {, ETP } \\
1570 \mathrm{~mm}, \mathrm{~T} 26.2^{\circ} \mathrm{C} \\
\text { Cálido seco, A } 120 \mathrm{~m}, \mathrm{P} 808 \mathrm{~mm} \text {, } \\
\text { ETP } 1621 \mathrm{~mm}, \mathrm{~T} 26.4^{\circ} \mathrm{C} \\
\text { Cálido, A } 25 \mathrm{~m}, \mathrm{P} 1216 \mathrm{~mm} \text {, ETP } \\
1739 \mathrm{~mm}, \mathrm{~T} 27^{\circ} \mathrm{C}\end{array}$ & $\begin{array}{l}\text { Profundos, bien drenados, texturas medias } \\
\text { a finas, fuertemente ácidos a alcalinos, alta } \\
\text { saturación de bases, fertilidad natural alta a } \\
\text { moderada }\end{array}$ \\
\hline Sur & $\begin{array}{l}\text { Guamal } \\
\text { Santa Bárbara } \\
\text { de Pinto }\end{array}$ & $\begin{array}{l}\text { Cálido húmedo, A } 25 \mathrm{~m}, \text { P } 1890 \mathrm{~mm} \text {, } \\
\text { ETP } 1757 \mathrm{~mm}, \mathrm{~T}^{\circ} 27.1^{\circ} \mathrm{C} \\
\text { Cálido seco, A } 22 \mathrm{~m}, \mathrm{P} 1400 \mathrm{~mm} \text {, } \\
\text { ETP } 1650 \mathrm{~mm}, \mathrm{~T} 28.5^{\circ} \mathrm{C}\end{array}$ & $\begin{array}{l}\text { Muy superficiales a profundos, bien } \\
\text { drenados, texturas finas a medias, } \\
\text { fuertemente ácidos a alcalinos, alta } \\
\text { saturación de bases, fertilidad natural baja } \\
\text { a alta }\end{array}$ \\
\hline
\end{tabular}

De acuerdo con las características notables de los elementos vegetales se definió la cobertura de árboles y cobertura de pastizales. La cobertura arbórea estuvo compuesta por plantas con elementos leñosos $\geq 4 \mathrm{~m}$ de altura, áreas de vegetación herbácea combinada con arbustos, distribuidos de manera relativamente homogénea y la cobertura de pastizales, plantas de contextura herbácea con elementos no leñosos, como pastos y hierbas (Londoño y Torres, 2015). Las muestras recolectadas fueron procesadas de conformidad con los estándares para la preparación de plantas desecadas.

\section{Inventario de especies}

La segunda etapa, fue descriptiva. Con la información básica, se identificó las especies mediante comparación con claves taxonómicas, la colección del herbario UTMC de la Universidad del Magdalena, bibliografía especializada y la consulta con expertos en el tema, siguiendo el sistema APG IV (Angiosperm Phylogeny Group). Los nombres de las familias y las especies se verificaron consultando The plant list (http://www.theplantlist.org/) y Tropicos (https://www.tropicos.org/home). Con la información anterior, se realizó el inventario de las especies del área de estudio, el hábito de crecimiento y los usos más comunes. Para esto, se tomó como referencia la metodología descrita por Londoño y Torres (2015) para clasificar las especies en arbustivas, arbóreas y hierbas terrestres, siguiendo su forma y tipo de crecimiento, se georreferenció la UP en la que fueron halladas y se procedió a caracterizarlas con su nombre común, científico, uso y aporte nutricional, priorizando aquellas que tienen potencial para ser incorporadas en sistemas agroforestales en el trópico seco colombiano. Para el estudio, se empleó el programa Excel producto de suscripción Microsoft office 365 Pro plus versión 1908 usando tablas dinámicas y gráficos. Los datos fueron analizados mediante estadística descriptiva y los gráficos elaborados con RAWGraphs (https://app.rawgraphs.io/).

\section{Análisis estadístico de la información}

Las diferencias en la riqueza de especies entre las UP muestreadas se determinaron mediante análisis de varianza unidireccional (ANOVA), probando primero los supuestos de aleatoriedad (prueba de Durbin Watson) y la normalidad de los residuos de ANOVA (prueba de Shapiro-Wilks) así como la homogeneidad de varianzas (prueba de Bartlett). Se evaluó la similitud de la composición florística entre las zonas según la presencia y ausencia de las especies, para lo que se realizó un análisis de conglomerados utilizando el coeficiente de Jaccard con el método de grupos de pares no ponderados con media aritmética (UPGMA) por presentar el mejor ajuste definido por su distancia cofonética. Estos análisis estadísticos se realizaron con el programa Rcmdr del software R 3.6.1 disponible en https://www.r-project.org/ 


\section{RESULTADOS}

En la Tabla 2 se muestran los resultados obtenidos por cada zona a partir del inventario de especies realizado en las unidades productivas.

\section{Riqueza y composición de especies}

De las 63 unidades productivas priorizadas en el proyecto Cambio Climático, se obtuvo el registro de 122 especies entre árboles, arbustos y hierbas terrestres que pertenecen a 18 familias diferentes (Tabla 2 y Figura 2).

Tabla 2: Inventario de especies recolectadas por unidad productiva (UP) municipio y zona - departamento del Magdalena.

\begin{tabular}{|c|c|c|}
\hline U. P & Pasturas (Nombre científico) & Especies arbóreas (Nombre científico) \\
\hline \multicolumn{3}{|r|}{ Zona sur } \\
\hline \multicolumn{3}{|r|}{ Municipio: Guamal } \\
\hline $\begin{array}{l}\text { Altos de San } \\
\text { José }\end{array}$ & $\begin{array}{l}\text { Panicum maximum, } \\
\text { Bothriochloa pertusa, } \\
\text { Pennisetum purpureum x } \\
\text { Pennisetum typhoides }\end{array}$ & $\begin{array}{l}\text { Tectona grandis, Swietenia Macrophylla, Moringa Oleífera, } \\
\text { Leucaena leucocephala, Gliricidia sepium }\end{array}$ \\
\hline Villa Tesca & Panicum máximum & $\begin{array}{l}\text { Albizia saman, Gliricidia sepium, Prosopis juliflora, Crescentia } \\
\text { cujete, Citrus sinensis }\end{array}$ \\
\hline $\begin{array}{l}\text { Plata Perdida- } \\
\text { La Admiración }\end{array}$ & $\begin{array}{l}\text { Dichantium aristatum, } \\
\text { Bothriochloa pertusa, } \\
\text { Cynodon nlemfuensis, } \\
\text { Panicum máximum } \\
\text { Pennisetum purpureum x } \\
\text { Pennisetum typhoides }\end{array}$ & $\begin{array}{l}\text { Guazuma ulmifolia, Spondias mombin, Albizia saman, Crescentia } \\
\text { cujete, Cedrela odorata, Tabebuia rosea, Tectona grandis, Citrus } \\
\text { sinensis }\end{array}$ \\
\hline $\begin{array}{l}\text { Perseverancia } \\
2\end{array}$ & $\begin{array}{l}\text { Bothriochloa pertusa, } \\
\text { Panicum máximum }\end{array}$ & $\begin{array}{l}\text { Gliricidia sepium, Crescentia cujete, Leucaena leucocephala, } \\
\text { Spondias mombin, Cordia alba, Guazuma ulmifolia, Albizia saman, } \\
\text { Citrus sinensis }\end{array}$ \\
\hline El Porvenir & $\begin{array}{l}\text { Panicum maximum, } \\
\text { Bothriochloa pertusa }\end{array}$ & $\begin{array}{l}\text { Albizia saman, Caesalpinia coriaria, Prosopis juliflora, Azadirachta } \\
\text { indica, Citrus sinensis }\end{array}$ \\
\hline Muzangal & $\begin{array}{l}\text { Panicum maximum, } \\
\text { Bothriochloa pertusa }\end{array}$ & $\begin{array}{l}\text { Tabebuia rosea, Gliricidia sepium, Mangifera indica, Guazuma } \\
\text { ulmifolia, Caesalpinia coriaria, Citrus sinensis }\end{array}$ \\
\hline San Antonio & $\begin{array}{l}\text { Digitaria decumbens, } \\
\text { Cynodon nlemfuensis }\end{array}$ & $\begin{array}{l}\text { Prosopis juliflora, Azadirachta indica, Guazuma ulmifolia, Albizia } \\
\text { saman }\end{array}$ \\
\hline San Jorge & $\begin{array}{l}\text { Panicum maximum, } \\
\text { Brachiaria brizantha }\end{array}$ & Albizia saman, Tabebuia rosea, Mangifera indica \\
\hline \multicolumn{3}{|c|}{ Municipio: Santa Barbara de Pinto } \\
\hline La Florida & $\begin{array}{l}\text { Bothriochloa pertusa, } \\
\text { Panicum maximum }\end{array}$ & Crescentia cujete, Guazuma ulmifolia, Gliricidia sepium, \\
\hline Centenario & $\begin{array}{l}\text { Bothriochloa pertusa } \\
\text { Panicum maximum }\end{array}$ & $\begin{array}{l}\text { Crescentia cujete, Guazuma ulmifolia, Prosopis juliflora, } \\
\text { Caesalpinia coriaria, Pereskia guamacho }\end{array}$ \\
\hline San Mateo & Bothriochloa pertusa & $\begin{array}{l}\text { Crescentia cujete, Caesalpinia coriaria, Guazuma ulmifolia, Albizia } \\
\text { saman }\end{array}$ \\
\hline La llusión & $\begin{array}{l}\text { Echinochloa polystachya, } \\
\text { Brachiaria brizantha, } \\
\text { Cynodon nlemfuensis }\end{array}$ & $\begin{array}{l}\text { Albizia saman, Guazuma ulmifolia, Crescentia cujete, Tabebuia } \\
\text { rosea }\end{array}$ \\
\hline La Divisa & Bothriochloa pertusa & Crescentia cujete, Prosopis juliflora, Guazuma ulmifolia \\
\hline Santa Rosa & Bothriochloa pertusa & $\begin{array}{l}\text { Spondias mombin, Crescentia cujete, Platymiscium pinnatum, } \\
\text { Caesalpinia coriaria, Acacia macracantha, Guazuma ulmifolia }\end{array}$ \\
\hline El Porvenir & $\begin{array}{l}\text { Bothriochloa pertusa, } \\
\text { Panicum maximum }\end{array}$ & $\begin{array}{l}\text { Crescentia cujete, Spondias mombin, Guazuma ulmifolia, Prosopis } \\
\text { juliflora }\end{array}$ \\
\hline El Tesoro & Bothriochloa pertusa & $\begin{array}{l}\text { Platymiscium pinnatum, Prosopis juliflora, Caesalpinia coriaria, } \\
\text { Albizia saman }\end{array}$ \\
\hline
\end{tabular}


Tabla 2: continuación.

\begin{tabular}{|c|c|c|}
\hline U. $P$ & Pasturas (Nombre científico) & Especies arbóreas (Nombre científico) \\
\hline \multicolumn{3}{|r|}{ Zona Centro } \\
\hline \multicolumn{3}{|r|}{ Municipio: Plato } \\
\hline Yoliseth & $\begin{array}{l}\text { Panicum maximum } \\
\text { Bothriochloa pertusa, } \\
\text { Brachiaria arrecta x } \\
\text { Brachiaria mutica, } \\
\text { Echinochloa polystachya, } \\
\text { Pennisetum purpureum, } \\
\text { Pennisetum purpureum x } \\
\text { Pennisetum typhoides, }\end{array}$ & $\begin{array}{l}\text { Enterolobium cyclocarpum, Albizia saman, Prosopis juliflora, } \\
\text { Acacia macracantha, Crescentia cujete, Platymiscium pinnatum, } \\
\text { Bulnesia arborea }\end{array}$ \\
\hline $\begin{array}{l}\text { Las Tres } \\
\text { Marías }\end{array}$ & $\begin{array}{l}\text { Panicum maximum, } \\
\text { Bothriochloa pertusa, } \\
\text { Brachiaria arrecta } x \\
\text { Brachiaria mutica }\end{array}$ & $\begin{array}{l}\text { Albizia saman, Platymiscium pinnatum, Azadirachta indica, } \\
\text { Prosopis juliflora, Crescentia cujete, Gliricidia sepium, }\end{array}$ \\
\hline El Olimpo & $\begin{array}{l}\text { Bothriochloa pertusa, } \\
\text { Panicum maximum } \\
\text { Digitaria decumbens, } \\
\text { Cynodon nlemfuensis, } \\
\text { Echinochloa polystachya, }\end{array}$ & $\begin{array}{l}\text { Guazuma ulmifolia, Crescentia cujete, Gliricidia sepium, Prosopis } \\
\text { juliflora, Acacia macracantha, Hymenaea courbaril, Platymiscium } \\
\text { pinnatum, }\end{array}$ \\
\hline Aguas Claras & $\begin{array}{l}\text { Brachiaria arrecta x } \\
\text { Brachiaria mutica, } \\
\text { Bothriochloa pertusa, } \\
\text { Echinochloa polystachya, } \\
\text { Cynodon nlemfuensis, } \\
\text { Dichantium aristatum, } \\
\text { Brachiaria mutica }\end{array}$ & $\begin{array}{l}\text { Albizia saman, Albizia niopoides, Platymiscium pinnatum, } \\
\text { Hymenaea courbaril, Enterolobium cyclocarpum, Ceiba pentandra, } \\
\text { Crescentia cujete, Moringa oleífera, Azadirachta indica, } \\
\text { Acacia macracantha, Prosopis juliflora }\end{array}$ \\
\hline La Patagonia & Bothriochloa pertusa & $\begin{array}{l}\text { Prosopis juliflora, Acacia macracantha, Platymiscium pinnatum, } \\
\text { Albizia saman, Cordia alba, Crescentia cujete, Guazuma ulmifolia, } \\
\text { Spondias mombin }\end{array}$ \\
\hline $\begin{array}{l}\text { Nueva } \\
\text { Esperanza }\end{array}$ & $\begin{array}{l}\text { Panicum maximum, } \\
\text { Bothriochloa pertusa }\end{array}$ & $\begin{array}{l}\text { Crescentia cujete, Bulnesia arborea, Guazuma ulmifolia, Gliricidia } \\
\text { sepium, Pereskia guamacho, Azadirachta indica, Spondias } \\
\text { mombin }\end{array}$ \\
\hline Los Deseos & $\begin{array}{l}\text { Bothriochloa pertusa, } \\
\text { Dichantium aristatum, } \\
\text { Cynodon nlemfuensis }\end{array}$ & $\begin{array}{l}\text { Crescentia cujete, Spondias mombin, Albizia saman, } \\
\text { Acacia macracantha, Prosopis juliflora, Gliricidia sepium, Bulnesia } \\
\text { arborea, Platymiscium pinnatum, Azadirachta indica }\end{array}$ \\
\hline La Bendición & $\begin{array}{l}\text { Panicum máximum, } \\
\text { Echinochloa polystachya }\end{array}$ & $\begin{array}{l}\text { Azadirachta indica, Mangifera indica, Platymiscium pinnatum, } \\
\text { Crescentia cujete, Manilkara zapota }\end{array}$ \\
\hline \multicolumn{3}{|c|}{ Municipio: Nueva Granada } \\
\hline La Mina & $\begin{array}{l}\text { Brachiaria arrecta } x \\
\text { Brachiaria mutica, } \\
\text { Bothriochloa pertusa, } \\
\text { Panicum maximum }\end{array}$ & $\begin{array}{l}\text { Crescentia cujete, Prosopis juliflora, Guazuma ulmifolia, } \\
\text { Enterolobium cyclocarpum }\end{array}$ \\
\hline Sinai 1 & $\begin{array}{l}\text { Bothriochloa pertusa, } \\
\text { Megathyrsus maximus, } \\
\text { Cynodon nlemfuensis }\end{array}$ & $\begin{array}{l}\text { Albizia saman, Bulnesia arborea, Prosopis juliflora, Spondias } \\
\text { mombin, Hymenaea courbaril, Platymiscium pinnatum, Crescentia } \\
\text { cujete }\end{array}$ \\
\hline Villa Edi & $\begin{array}{l}\text { Bothriochloa pertusa, } \\
\text { Brachiaria arrecta } x \\
\text { Brachiaria mutica, } \\
\text { Megathyrsus maximus, } \\
\text { Panicum maximum, } \\
\text { Pennicetum purpureum }\end{array}$ & $\begin{array}{l}\text { Platymiscium pinnatum, Prosopis juliflora, Acacia macracantha, } \\
\text { Crescentia cujete, Enterolobium cyclocarpum, Handroanthus } \\
\text { billbergii }\end{array}$ \\
\hline Voy a Ver & $\begin{array}{l}\text { Bothriochloa pertusa, } \\
\text { Megathyrsus maximus. }\end{array}$ & $\begin{array}{l}\text { Albizia saman, Gliricidia sepium, Platymiscium pinnatum, } \\
\text { Handroanthus billbergii, Roseodendron chryseum }\end{array}$ \\
\hline La Carpa & $\begin{array}{l}\text { Bothriochloa pertusa, } \\
\text { Panicum máximum, } \\
\text { Pennisetum purpureum }\end{array}$ & $\begin{array}{l}\text { Crescentia cujete, Azadirachta indica, Prosopis juliflora, } \\
\text { Platymiscium pinnatum, Albizia saman, Guazuma ulmifolia, } \\
\text { Bulnesia arborea, Gliricidia sepium, Spondias mombin, } \\
\text { Enterolobium cyclocarpum . }\end{array}$ \\
\hline
\end{tabular}


Tabla 2: continuación.

\begin{tabular}{|c|c|c|}
\hline U. $P$ & Pasturas (Nombre científico) & Especies arbóreas (Nombre científico) \\
\hline Guayaquil & $\begin{array}{l}\text { Bothriochloa pertusa, Panicum } \\
\text { maximum, Brachiaria arrecta x } \\
\text { Brachiaria mutica, Echinochloa } \\
\text { polystachya }\end{array}$ & $\begin{array}{l}\text { Enterolobium cyclocarpum, Spondias mombin, Bulnesia arborea, } \\
\text { Caesalpinia coriaria, Aspidosperma polyneuron, Crescentia cujete, } \\
\text { Guazuma ulmifolia, Albizia saman, Azadirachta indica }\end{array}$ \\
\hline Nazaret & $\begin{array}{l}\text { Bothriochloa pertusa, } \\
\text { Brachiaria arrecta } \times \text { Brachiaria } \\
\text { mutica, Panicum maximum }\end{array}$ & $\begin{array}{l}\text { Crescentia cujete, Guazuma ulmifolia, Handroanthus billbergii, } \\
\text { Acacia macracantha, Sterculia apetala, Bulnesia arborea }\end{array}$ \\
\hline El Rosario & $\begin{array}{l}\text { Bothriochloa pertusa, } \\
\text { Megathyrsus maximus, } \\
\text { Brachiaria arrecta x Brachiaria } \\
\text { mutica, Panicum maximum, } \\
\text { Pennisetum purpureum }\end{array}$ & $\begin{array}{l}\text { Acacia macracantha, Prosopis juliflora, Azadirachta indica, } \\
\text { Platymiscium pinnatum, Tabebuia rosea, Handroanthus billbergii, } \\
\text { Crescentia cujete, Bulnesia arborea, Moringa oleífera, Guazuma } \\
\text { ulmifolia }\end{array}$ \\
\hline \multicolumn{3}{|r|}{ Municipio: Chibolo } \\
\hline $\begin{array}{l}\text { San Juan de } \\
\text { Tadeo }\end{array}$ & Bothriochloa pertusa & $\begin{array}{l}\text { Prosopis juliflora, Acacia macracantha, Albizia saman, Gliricidia } \\
\text { sepium, Crescentia cujete }\end{array}$ \\
\hline $\begin{array}{l}\text { Santa Inés del } \\
\text { Monte }\end{array}$ & $\begin{array}{l}\text { Bothriochloa pertusa, } \\
\text { Brachiaria arrecta x Brachiaria } \\
\text { mutica }\end{array}$ & $\begin{array}{l}\text { Albizia saman, Malpighia glabra, Prosopis juliflora, Cordia alba, } \\
\text { Gliricidia sepium, Azadirachta indica, Quadrella odoratissima }\end{array}$ \\
\hline San Diego & $\begin{array}{l}\text { Bothriochloa pertusa, Panicum } \\
\text { maximum }\end{array}$ & $\begin{array}{l}\text { Albizia saman, Tabebuia rosea, Azadirachta indica, Prosopis } \\
\text { juliflora, Hevea brasiliensis, Crescentia cujete, Enterolobium } \\
\text { cyclocarpum, Hymenaea courbaril }\end{array}$ \\
\hline Vallan Viendo & $\begin{array}{l}\text { Brachiaria arrecta x Brachiaria } \\
\text { mutica }\end{array}$ & Azadirachta indica, Crescentia cujete, Gliricidia sepium \\
\hline California & $\begin{array}{l}\text { Bothriochloa pertusa, Panicum } \\
\text { maximum }\end{array}$ & $\begin{array}{l}\text { Albizia saman, Enterolobium cyclocarpum, Azadirachta indica, } \\
\text { Gliricidia sepium, Miroxylon balsamun, }\end{array}$ \\
\hline Panorama & $\begin{array}{l}\text { Bothriochloa pertusa, } \\
\text { Brachiaria arrecta x Brachiaria } \\
\text { mutica, Brachiaria mutica, } \\
\text { Cynodon nlemfuensis }\end{array}$ & $\begin{array}{l}\text { Albizia saman, Prosopis juliflora, Tabebuia rosea, Quadrella } \\
\text { odoratissima, Bulnesia arborea, Platymiscium pinnatum, } \\
\text { Acacia macracantha, Gliricidia sepium. }\end{array}$ \\
\hline El Diamante & $\begin{array}{l}\text { Bothriochloa pertusa, } \\
\text { Dichantium aristatum benth, } \\
\text { Brachiaria arrecta x Brachiaria } \\
\text { mutica, Panicum maximum } \\
\text { Cynodon nlemfuensis }\end{array}$ & $\begin{array}{l}\text { Prosopis juliflora, Enterolobium cyclocarpum, Sterculia apetala, } \\
\text { Lecythis minor, Handroanthus billbergii, Spondias mombin }\end{array}$ \\
\hline Las Colinas & $\begin{array}{l}\text { Bothriochloa pertusa, Panicum } \\
\text { maximum, Brachiaria mutica }\end{array}$ & $\begin{array}{l}\text { Crescentia cujete, Albizia saman, Enterolobium cyclocarpum, } \\
\text { Azadirachta indica, Prosopis juliflora, Spondias mombin, Guazuma } \\
\text { ulmifolia }\end{array}$ \\
\hline \multicolumn{3}{|c|}{ Municipio: Sabanas de San Ángel } \\
\hline Tallahassee & $\begin{array}{l}\text { Megathyrsus maximus, } \\
\text { Bothriochloa pertusa }\end{array}$ & $\begin{array}{l}\text { Albizia saman, Crescentia cujete, Guazuma ulmifolia, Prosopis } \\
\text { juliflora, Hymenaea courbaril, Spondias mombin }\end{array}$ \\
\hline Los Rosales & $\begin{array}{l}\text { Panicum maximum } \\
\text { Bothriochloa pertusa, } \\
\text { Pennicetum purpureum }\end{array}$ & $\begin{array}{l}\text { Crescentia cujete, Gliricidia sepium, Guazuma ulmifolia, Albizia } \\
\text { saman, Moringa oleífera, Spondias mombin }\end{array}$ \\
\hline $\begin{array}{l}\text { Santa } \\
\text { Catalina }\end{array}$ & $\begin{array}{l}\text { Panicum maximum, Cynodon } \\
\text { nlemfuensis, Bothriochloa } \\
\text { pertusa }\end{array}$ & $\begin{array}{l}\text { Albizia saman, Enterolobium cyclocarpum, Sterculia apetala, Acacia } \\
\text { melanoxylon, Guazuma ulmifolia, Gliricidia sepium, Crescentia } \\
\text { cujete }\end{array}$ \\
\hline Lloraito & $\begin{array}{l}\text { Megathyrsus maximus, } \\
\text { Bothriochloa pertusa, Panicum } \\
\text { maximum, Andropogon } \\
\text { gayanus }\end{array}$ & $\begin{array}{l}\text { Tabebuia rosea, Platymiscium pinnatum, Bulnesia arborea, } \\
\text { Guazuma ulmifolia, Gliricidia sepium, Spondias mombin, Prosopis } \\
\text { juliflora, Albizia saman, Crescentia cujete. }\end{array}$ \\
\hline Corral Nuevo & $\begin{array}{l}\text { Panicum maximum, } \\
\text { Bothriochloa pertusa } \\
\end{array}$ & Prosopis juliflora, Handroanthus billbergii, Crescentia cujete \\
\hline Risaralda & $\begin{array}{l}\text { Megathyrsus maximus, } \\
\text { Bothriochloa pertusa }\end{array}$ & $\begin{array}{l}\text { Bulnesia arborea, Gliricidia sepium, Guazuma ulmifolia, Crescentia } \\
\text { cujete, Spondias mombin }\end{array}$ \\
\hline San Cayetano & Panicum máximum & $\begin{array}{l}\text { Bulnesia arborea, Albizia saman, Prosopis juliflora, Guazuma } \\
\text { ulmifolia, Crescentia cujete }\end{array}$ \\
\hline $\begin{array}{l}\text { Los } \\
\text { Recuerdos }\end{array}$ & $\begin{array}{l}\text { Bothriochloa pertusa, Panicum } \\
\text { maximum }\end{array}$ & \\
\hline
\end{tabular}


Tabla 2: continuación.

\begin{tabular}{|c|c|c|}
\hline \multicolumn{3}{|r|}{ Zona Río } \\
\hline \multicolumn{3}{|r|}{ Municipio: Sitio Nuevo } \\
\hline La Sonrisa & $\begin{array}{l}\text { Cynodon nlemfuensis, Panicum } \\
\text { maximum, Echinochloa } \\
\text { polystachya, }\end{array}$ & Quadrella odoratissima, Prosopis juliflora \\
\hline SamPayo & Cynodon nlemfuensis & $\begin{array}{l}\text { Albizia saman, Prosopis juliflora, Guazuma ulmifolia, Quadrella } \\
\text { odoratissima, Acacia macracantha }\end{array}$ \\
\hline Villa Paola & $\begin{array}{l}\text { Echinochloa polystachya, } \\
\text { Brachiaria Arrecta x Brachiaria } \\
\text { mutica, Cynodon nlemfuensis, } \\
\text { Bothriochloa pertusa }\end{array}$ & $\begin{array}{l}\text { Tabebuia rosea, Albizia saman, Gliricidia sepium, Prosopis juliflora, } \\
\text { Guazuma ulmifolia, Acacia macracantha, Platymiscium pinnatum }\end{array}$ \\
\hline Monterrey & $\begin{array}{l}\text { Echinochloa polystachya, } \\
\text { Cynodon nlemfuensis, } \\
\text { Brachiaria mutica, Panicum } \\
\text { maximum }\end{array}$ & $\begin{array}{l}\text { Albizia saman, Cordia alba, Azadirachta indica, Guazuma ulmifolia, } \\
\text { Gliricidia sepium, Tabebuia rosea, Spondias mombin, Prosopis } \\
\text { juliflora, Mangifera indica }\end{array}$ \\
\hline San Miguel & $\begin{array}{l}\text { Panicum maximum, Brachiaria } \\
\text { mutica }\end{array}$ & $\begin{array}{l}\text { Albizia saman, Gliricidia sepium, Tabebuia rosea, Mangifera indica, } \\
\text { Cordia alba, Guazuma ulmifolia, Quadrella odoratissima. }\end{array}$ \\
\hline San Rafael & $\begin{array}{l}\text { Brachiaria mutica, Cynodon } \\
\text { nlemfuensis }\end{array}$ & $\begin{array}{l}\text { Albizia saman, Tabebuia rosea, Guazuma ulmifolia, Spondias } \\
\text { mombin, Prosopis juliflora, Cordia alba, Azadirachta indica, } \\
\text { Crescentia cujete }\end{array}$ \\
\hline La conquista & Dichantium aristatum & $\begin{array}{l}\text { Quadrella odoratissima, Prosopis juliflora, Cordia alba, Guazuma } \\
\text { ulmifolia, Azadirachta indica }\end{array}$ \\
\hline \multicolumn{3}{|r|}{ Zona Norte } \\
\hline \multicolumn{3}{|c|}{ Municipio: Zona Bananera } \\
\hline Padelma & & Musa paradisiaca \\
\hline Pa los dos & Bothriochloa pertusa & $\begin{array}{l}\text { Mangifera indica, Crescentia cujete, Albizia saman, Sterculia } \\
\text { apetala, Chloroleucon mangense }\end{array}$ \\
\hline Mi Esfuerzo & Bothriochloa pertusa & $\begin{array}{l}\text { Mangifera indica, Crescentia cujete, Albizia saman, Sterculia } \\
\text { apetala, Chloroleucon mangense }\end{array}$ \\
\hline Las Delicias & & Musa paradisiaca \\
\hline Chile Gaira & Saccharum officinarum & Mangifera indica \\
\hline La Cecilia & & Musa paradisiaca \\
\hline Tierra Nueva & Pennisetum purpureum & Albizia saman \\
\hline
\end{tabular}

Se encontraron diferencias $(p<0,05)$ en la riqueza de especies entre las zonas evaluadas, siendo Centro, la zona con mayor número de especies, seguido de la zona Sur, mientras que las zonas Norte y Río mostraron los menores valores en la variable (Figura 2). Las familias con mayor riqueza de especies fueron Poaceae (20), Fabaceae (13), Bignoniaceae (4), Meliaceae (3) seguidas por Anacardiaceae y Malvaceae con 2 especies cada una, las demás familias presentes suman 13 especies. Una vez se realiza el inventario de especies se inicia la clasificación según lo descrito por Londoño y Torres (2015) (Tablas 3 y 4) y se incorpora el uso a partir de los datos reportados por los productores en la zona y la información secundaria.

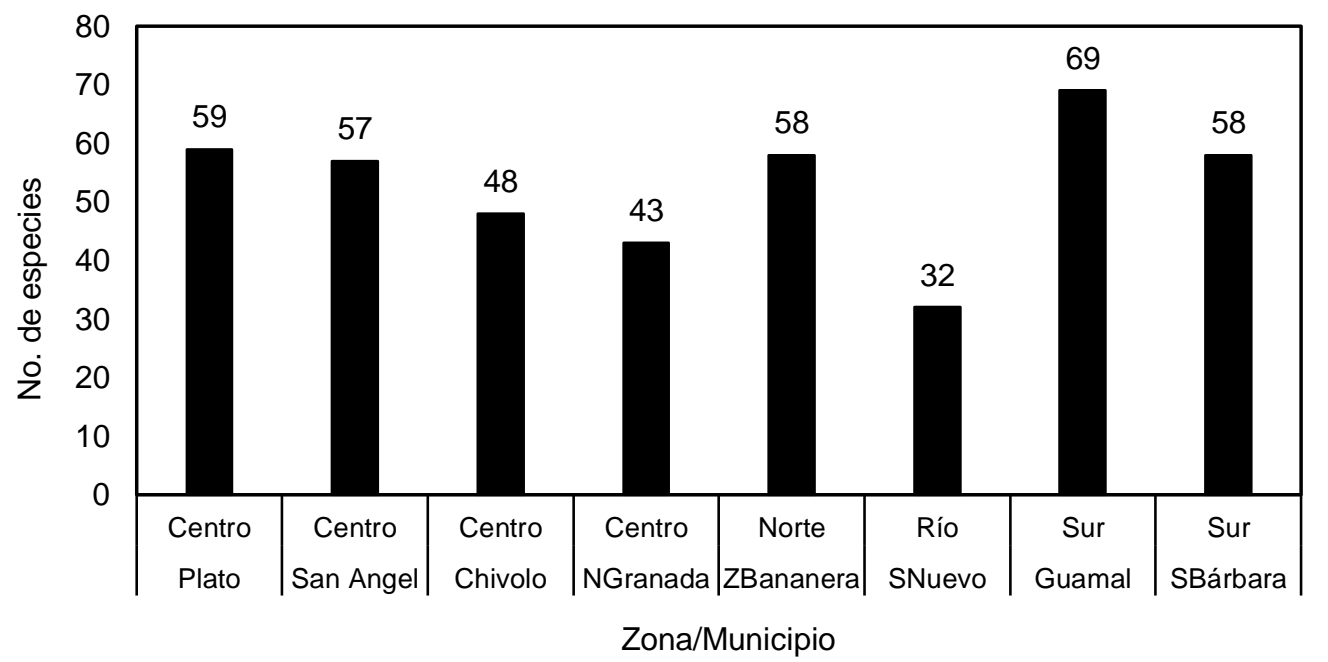

Fig. 2: Número de especies encontradas en cada uno de los municipios muestreados. 
Tabla 3: Clasificación y uso de las especies de árboles y arbustos encontrados en 63 UP del departamento del Magdalena.

\begin{tabular}{|c|c|c|c|c|}
\hline Especie (Nombre científico) & $\begin{array}{l}\text { Nombre } \\
\text { Común }\end{array}$ & Familia & Usos & Clasificación \\
\hline Gliricidia sepium & Matarratón & Fabaceae & Cerca viva/Forraje/ Mielifera & Árbol \\
\hline Mangifera indica & Mango & Anacardiaceae & Comestible & Árbol \\
\hline Manilkara zapota & Níspero & Sapotaceae & Comestible & Árbol \\
\hline Psidium guajava & Guayaba & Myrtaceae & Comestible & Árbol \\
\hline Guazuma ulmifolia & Guácimo & Malvaceae & Maderable & Árbol \\
\hline Citrus sinensis & Naranja & Rutaceae & Comestible & Árbol \\
\hline Caesalpinia coriaria & dividive & Fabaceae & Maderable & Árbol \\
\hline Acacia melanoxylon & Acacia & Fabaceae & Construcción/Cerca viva & Árbol \\
\hline Azadirachta indica & Neem & Meliaceae & construcción/Ornamental & Árbol \\
\hline Spondias mombin & Hobo & Anacardiaceae & Maderable & Árbol \\
\hline Cordia alba & Uvito & Boraginaceae & Maderable & Árbol \\
\hline Crescentia cujete & Totumo & Bignoniaceae & Forraje/Fruto para ganado & Árbol \\
\hline Cedrela odorata & Cedro & Meliaceae & Maderable / Medicinal & Árbol \\
\hline Ceiba pentandra & Bonga & Bombacaeae & Maderable/Forraje & Árbol \\
\hline Prosopis juliflora & Trupillo & Fabaceae & Maderable/forraje & Árbol \\
\hline Enterolobium cyclocarpum & Orejero & Fabaceae & Maderable/Fruto para ganado & Árbol \\
\hline Miroxylon balsamun & Bálsamo & Fabaceae & Maderable/Ornamental & Árbol \\
\hline Sterculia apetala & Camajon & Malvaceae & Maderable/Ornamental & Árbol \\
\hline Roseodendron chryseum & Puy & Bignoniaceae & Maderable/Ornamental & Árbol \\
\hline Hevea Brasiliensis & Caucho & Euphorbiaceae & Maderable/Ornamental & Árbol \\
\hline Albizia niopoides & $\begin{array}{l}\text { Guacamay } \\
\text { o }\end{array}$ & Fabaceae & Maderable/Ornamental & Árbol \\
\hline Handroanthus billbergii & Lumbre & Bignoniaceae & Maderable & Árbol \\
\hline Tabebuia rosea & Roble & Bignoniaceae & Maderable/Ornamental & Árbol \\
\hline Platymiscium pinnatum & Trebol & Fabaceae & Maderable/Ornamental & Árbol \\
\hline Hymenaea courbaril & Algarrobo & Fabaceae & Maderable/Ornamental & Árbol \\
\hline Albizia saman & Campano & Fabaceae & Maderable/Ornamental & Árbol \\
\hline Bulnesia arbórea & Guayacán & Zygophyllaceae & Maderable/Ornamental & Árbol \\
\hline Tectona grandis & Teca & Verbenaceae & Maderable/Ornamental & Árbol \\
\hline Swietenia macrophylla & Caoba & Meliaceae & Maderable/Ornamental & Árbol \\
\hline Moringa oleífera & Moringa & Moringaceae & Medicinal & Árbol \\
\hline Pereskia guamacho & Guamacho & Cactaceae & Ornamental & Árbol \\
\hline Malpighia glabra & Cerezo & Malpighiaceae & Comestible/Ornamental & Arbusto \\
\hline Acacia macracantha & Aromo & Fabaceae & Maderable/Forraje & Arbusto \\
\hline Quadrella odoratissima & Olivo & Capparaceae & Ornamental & Arbusto \\
\hline
\end{tabular}

Tabla 4: Clasificación y uso de las especies de hierbas encontradas en 63 UP del departamento del Magdalena.

\begin{tabular}{|c|c|c|c|c|}
\hline Especie (Nombre científico) & Nombre Común & Familia & Usos & Clasificación \\
\hline Brachiaria mutica & Admirable & Poaceae & Forraje & Hierba \\
\hline Echinochloa polystachya & Pasto alemán & Poaceae & Forraje & Hierba \\
\hline Dichantium aristatum & Angleton & Poaceae & Forraje & Hierba \\
\hline Brachiaria $s p$ & Brachiaria & Poaceae & Forraje & Hierba \\
\hline B. arrecta $\times$ B. mutica & Brachipara & Poaceae & Forraje & Hierba \\
\hline Bothriochloa pertusa & Colosuana & Poaceae & Forraje & Hierba \\
\hline Cynodon nlemfuensis & Pasto estrella & Poaceae & Forraje & Hierba \\
\hline Panicum máximum & Guinea mombasa & Poaceae & Forraje & Hierba \\
\hline $\begin{array}{llll}\begin{array}{l}\text { Pennisetum } \\
\text { typhoides }\end{array} & \text { purpureum } & \text { y } & P . \\
\end{array}$ & King grass & Poaceae & Forraje & Hierba \\
\hline Pennisetum purpureum & Pasto elefante & Poaceae & Forraje & Hierba \\
\hline Digitaria decumbens & Pasto pangola & Poaceae & Forraje & Hierba \\
\hline Megathyrsus maximus & Guinea tanzania & Poaceae & Forraje & Hierba \\
\hline Brachiaria brizantha & Pasto toledo & Poaceae & Forraje & Hierba \\
\hline
\end{tabular}


El $66 \%$ de las especies presentes en las unidades productivas seleccionadas fueron clasificadas según su forma de crecimiento como árboles, es decir, individuos con crecimiento secundario y acumulación de tejido leñoso que forman un tallo o fuste definido y que alcanzan la madurez a una altura mayor o igual a $4 \mathrm{~m}$ (Londoño y Torres, 2015). El $28 \%$ de las especies fueron clasificadas como hierbas terrestres debido a que son plantas sin crecimiento secundario, o poco aparente, en general con porte pequeño que crecen sobre el suelo, además no se clasifican como trepadoras, hemiepífitas o epífitas. El $6 \%$ de las especies encontradas fueron plantas leñosas $\sin$ tronco claramente definido, muy ramificadas desde la base del tallo que alcanzan la madurez a una altura menor que $4 \mathrm{~m}$, por lo que fueron clasificadas como arbustos. En las tablas 3 y 4 , se observan las especies reportadas en las 63 unidades productivas con su respectivo nombre científico, familia, usos y clasificación.

Se encontró en total 18 familias. La Figura 3 muestra el porcentaje de plantas por familia, donde se verificó el predominio de Poáceas y Fabáceas. El $27 \%$ de las especies fueron asignadas a la familia Poaceae, seguido de Fabaceae con el $25 \%$, Bignoniaceae con $8.3 \%$, Meliaceae con $6.3 \%$, Anacardiaceae y Malvaceae con $4.26 \%$ cada una. El resto de las familias representaron solo el $2.1 \%$ cada una. La familia Poaceae se encontró en mayor porcentaje en las UP del estudio en las que se dedican a la actividad ganadera manejada bajo el sistema doble propósito, por lo que cuentan con una gran cantidad de hierbas terrestres o pastos que sirven como alimento para el ganado. Por su parte las Fabáceas también pueden ser usadas como plantas forrajeras, de hecho, el $29 \%$ de las especies estudiadas tienen este uso, sin embargo, la mayoría de las especies en estudio fueron clasificadas como maderables y representaron el $35 \%$, en concordancia con lo encontrado por Lokonon et al. (2019) quienes afirman que entre los agricultores existe una marcada preferencia por las especies que brindan madera, energía, alimento y medicina. Las plantas ornamentales alcanzaron $25 \%$ teniendo en cuenta que muchas de ellas, tienen más de un uso.

Por otro lado, se evidenció con diferencias significativas $(P<0,05)$, que la Zona centro mostró el mayor número de familias y especies, seguido de la Zona Sur y la Zona Norte (Figura 4). Después de analizar cada una de las especies encontradas en las zonas de estudio se priorizaron las que tienen potencial para sistemas agroforestales. Villanueva-López et al. (2019) enuncian que especies arbustivas y arbóreas que cumplan con funciones como: sombra, cercas vivas, forrajes, bancos de proteína, medicinales, ornamentales, protectoras de fuentes de agua y mejoradoras de las propiedades físicas, químicas y biológicas de los suelos deben seleccionarse. De acuerdo a lo anterior, y respecto al uso, se estableció la representación del porcentaje de uso encontrándose $35 \%$ de maderables, $29 \%$ de forrajes, $25 \%$ ornamentales, $3 \%$ de medicinales, cercas vivas y construcción y $2 \%$ que pertenecen a las melíferas.

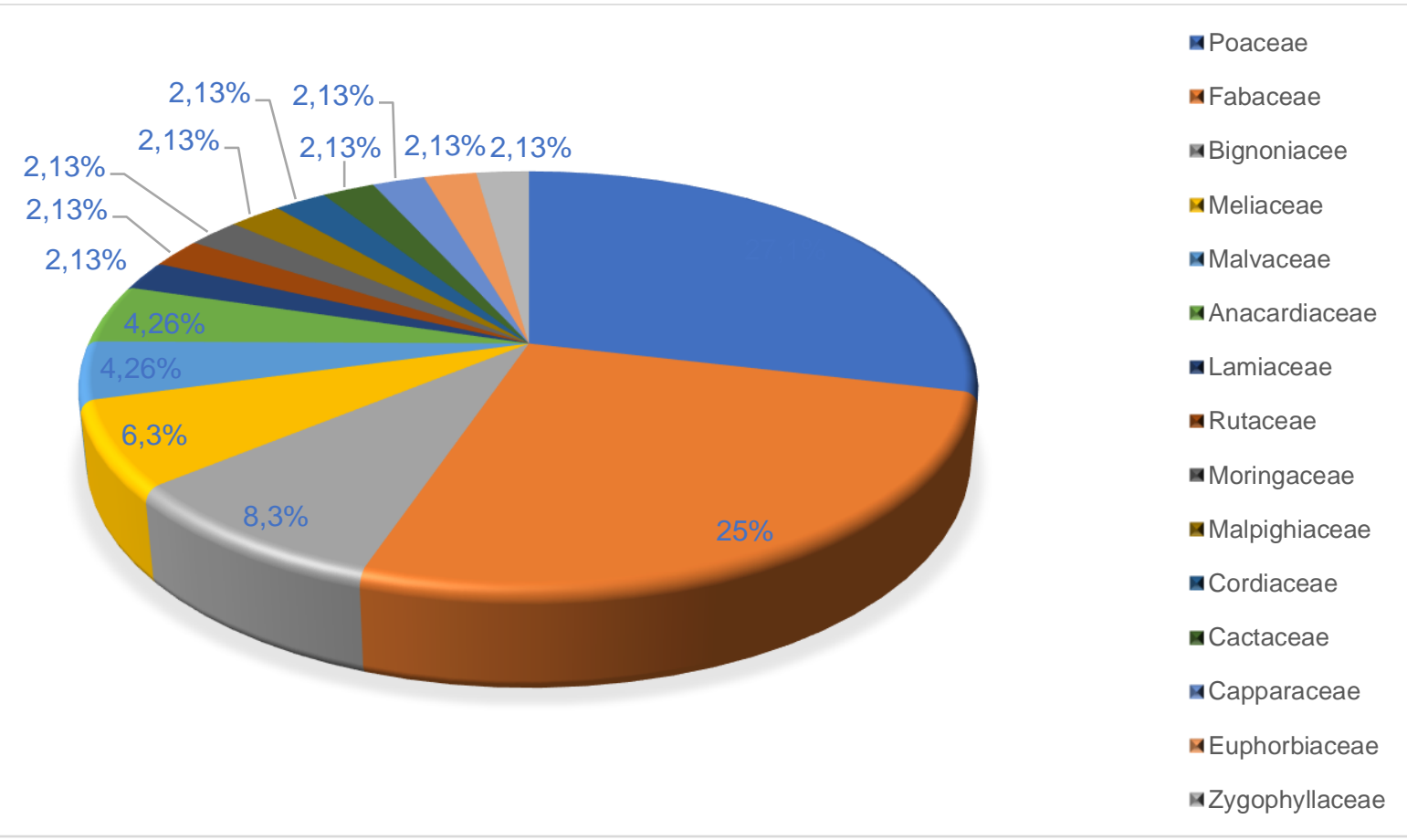

Fig. 3: Porcentaje de especies por familia encontradas en el Departamento del Magdalena. 
El análisis multivariado mostró que las áreas de estudio se asociaron en tres grupos de acuerdo al grado de similaridad en la composición florística (Figura 5). El primer grupo lo conformó precisamente la Zona centro (con mayor número de especies y familias), el segundo grupo lo conformaron las Zonas Norte y Sur y el tercer grupo lo conformó la Zona Río (con el menor número de especies) observándose amplia diferencia con los otros grupos. Además, se encontró diferencias entre las zonas en términos de composición florística entre las zonas Centro, Río y Norte-Sur. Las discrepancias entre UP, también fueron evidentes. La zona Norte-Sur presentó la más alta disimilaridad (gran distancia entre centroides), seguida de la zona Centro, mientras que la zona Río presentó alta similaridad. Lo anterior indica que existen especies que se adaptaron a las condiciones agroclimáticas de cada zona.

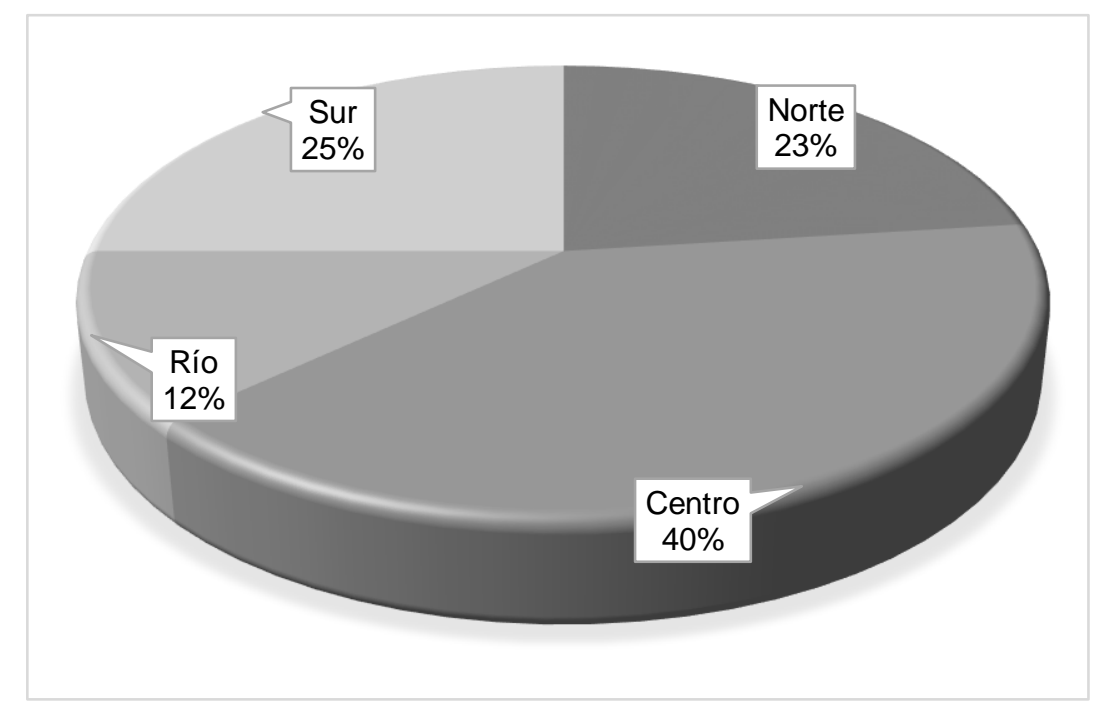

Fig. 4: Distribución de familias vegetales encontradas en las Unidades Productivas con potencial para sistemas agroforestales.

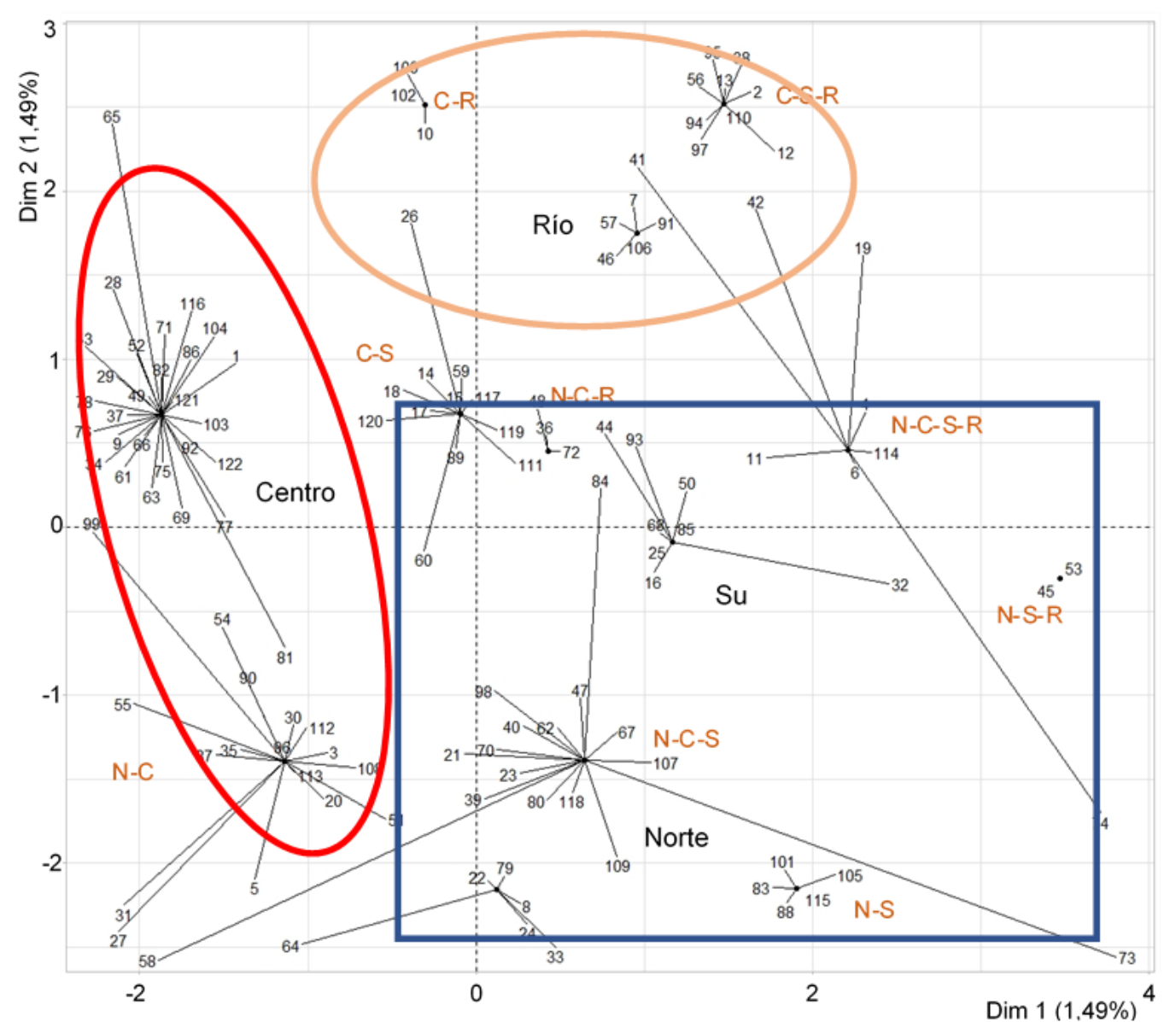

Fig. 5: Agrupamiento de las zonas de estudio y ordenación en la composición florística, de acuerdo a la similaridad (distancia entre centroides) en las comunidades vegetales. 


\section{DISCUSIÓN}

El sistema ganadero que predomina en el departamento del Magdalena es extensivo, los bovinos recorren grandes distancias en búsqueda de alimento, la cobertura forestal observada en las UP fue baja, grandes potreros con pocas especies forestales. Los productores reconocen que han deforestado para adquirir leña 0 ampliar el potrero, según afirma uno de los entrevistados. "Se tala y se quema para abrir espacio y crear un potrero limpio" situación que es amenaza ante la vulnerabilidad ambiental del territorio (Revueltas et al., 2020). Sin embargo, Iglesias Gómez, et al. (2019) y Buitrago-Guillen et al. (2018) afirman que, relictos pequeños de bosque 0 arbustos solitarios en los potreros evidencian la pertinencia de incluir sistemas agroforestales al observar que el ganado busca sombra y el forraje es más fresco en su cercanía.

\section{Especies de pasturas dominantes}

Hacia el centro y sur del departamento, en las UP muestreadas se evidenció la presencia de forrajes como la Bothriochloa pertusa, que abunda respecto a otras especies, probablemente por las características de colonización. Autores como Khan et al. (2019) la consideran gramínea invasora que desplaza especies nativas, se reproduce por semillas y estolones, y es resistente al pisoteo del ganado, con una amplia capacidad adaptativa. Además, argumentan que tiene efecto alelopático que inhibe germinación y crecimiento de otras especies y presenta asociación con micorrizas arbusculares, lo que le brinda ventajas para su establecimiento. Hacia el norte del departamento, en el municipio Sitio Nuevo, las especies predominantes son Cynodon nlemfuensis (tolerante a inundaciones) y Echinochloa polystachya (presente en zonas pantanosas u orillas de ríos y complejos cenagosos) coincidiendo con la descripción de Díaz-Céspedes et al. (2020) quienes afirman que las mejores repuestas en productividad se presentan en zonas húmedas y se comporta bien en condiciones de inundación. Así mismo, se encontró gramíneas que crecen de forma natural, donde sobresalen Bothriochloa pertusa como una especie invasora en el cultivo de banano y Dichanthium aristatum (Quintero-Pertúz et al., 2020).

\section{Especies arbóreas}

Hacia el centro y norte del departamento se observó presencia de especies en regeneración como Prosopis juliflora, Crescentia cujete, Samanea saman y Chloroleucon mangense, especialmente útiles en los sistemas de producción ganadera ya que producen semillas y hojas que sirven de alimento al ganado, incluso mejoran el perfil nutricional de los forrajes convencionales al aportar proteínas y otros nutrientes esenciales (Shiferaw et al., 2019; Lokonon et al., 2019; Romero-Duque et al., 2007). Hacia el sur del departamento, la mayoría de las UP muestreadas evidencian más cobertura forestal respecto al norte y centro, dos UP han incorporado prácticas del modelo agroforestal. Se encontró arbustos y árboles en mayor proporción y sobresalen especies como Crescentia cujete, Guazuma ulmifolia, Prosopis juliflora, Chloroleucon mangense, Platymisium pinnatum, Samanea Samán, Manilkara zapota, Hymenaea courbaril, Gliricidia sepium y Cedrela odorata, entre otros. Sin embargo, hay evidencias que permiten deducir que la ampliación de la frontera agropecuaria, erradicó muchas especies nativas y alteró las condiciones de los ecosistemas (Lokonon et al., 2019), prácticas que homogenizan el paisaje.

Precisamente, Revueltas et al. (2020) y Buitrago-Guillen et al. (2018) enuncian que, entre más diversos sean los agroecosistemas, mayor será su resiliencia ecológica y la posibilidad de adaptación a escenarios cambiantes, es importante que el productor reconozca la prioridad implementar prácticas de conservación y protección a los recursos naturales en su finca, el uso de sistemas agro - silvopastoriles (bancos forrajeros, cercas vivas, barreras cortavientos, cosecha de frutos y follaje, incorporación de BPG, entre otras prácticas) como alternativa de producción que mejora el flujo de la materia orgánica e incorpora carbono al suelo estabilizando ciclos biogeoquímicos.

Resultados de investigaciones de Mancera et al. (2018) y Buitrago-Guillen et al. (2018), respecto a sistemas agroforestales, evidencian la pertinencia de su incorporación en el trópico, al fomentar la reestructuración vegetal de los agro ecosistemas, donde los árboles y arbustos favorecen poblaciones de aves e insectos, suministran sombra, realizan fijación biológica de nitrógeno, sirven de alimento entre otros, lo que incrementa la producción bovina al reducir el estrés de los animales. Adicionalmente, los mismos autores enuncian que la introducción de leguminosas en los sistemas silvopastoriles, mejoran la digestibilidad de las dietas y la respuesta productiva. Ríos et al., (2006) trabajando en Nicaragua y Costa Rica, registraron que la escorrentía fue significativamente más alta en pasturas degradadas (42\%) comparados con los bancos forrajeros con leñosas perennes (3\%), bosques secundarios jóvenes $(6 \%)$ y las pasturas con alta densidad de árboles (12 $\%)$, evidencia que pone de manifiesto que el uso de la cobertura vegetal y arbórea en las condiciones donde se realizó el estudio, es altamente benéfico no solo para la retención de agua, sino que además incrementa la fijación de Nitrógeno y reduce las pérdidas de suelo por erosión (Revueltas et al.,2020). 
Así mismo, Zabala et al. (2018) reportaron que, en pasturas, el carbono en el suelo se incrementa. Sin embargo, los sistemas de raíces de las mismas, están concentrados en los estratos superiores y hay poco en las capas más profundas. Los mismos autores mencionan que los sistemas agroforestales secuestran carbono en los suelos además de incorporarlo en la biomasa leñosa. El carbono total en los sistemas silvopastoriles (SSP) varía entre $89-285 \mathrm{t} \mathrm{ha}^{-1}$. Otros estudios argumentan la capacidad de los SSP para reducir las emisiones de $\mathrm{CH}_{4}$ por fermentación entérica, tanto in vivo (Molina et al., 2016) como in vitro y la medición de los flujos de gases provenientes de praderas y excreciones bovinas. Así mismo, el uso de sistemas silvopastoriles en la ceba de ganado permitió obtener ganancias promedio diarias por animal entre 400 y $600 \mathrm{~g}$ y se incorpora la hembra a la reproducción entre los 23 y 25 meses, evidenciando incremento respecto al sistema tradicional. Por otro lado, después de más de nueve años de estudio en SSP, Ramakrishnan et al. (2020) concluyeron que cumplen un papel importante, en la mejora de la productividad e ingresos en regiones semiáridas, protegen el suelo y de la degradación y son vitales para los ecosistemas de regiones semiáridas. En la Tabla 5, se resumen las especies encontradas de forma dispersas en potreros y algunos relictos de la zona de estudio, especies reconocidas por su potencial para ser incorporadas a sistemas agroforestales y pueden ser referentes para modelos a implementar en la zona.

Lo anterior evidencia que existen investigaciones que respaldan la incorporación de las especies encontradas al sistema, conocimiento que viabiliza la alternativa seleccionada. Dada la producción de biomasa, composición química, aporte nutricional, sombrío, albergue de fauna y flora silvestre (Mancera et al., 2018), además de algunos reportes como recuperadoras de suelo, por la capacidad de fijar nitrógeno, al presentar en sus raíces asociación con bacterias del género Rhizobium; o con micorrizas, evidencian la pertinencia desde el punto de vista ecológico y ratifican la implementación de los sistemas agroforestales como alternativa del sector agropecuario en la zona de estudio ante cambio climático. Pero también, es incuestionable que las condiciones geológicas, climáticas y de diversidad de suelos existentes en el Departamento determinan la comunidad de especies que se establecen (Quintero-Pertúz et al., 2020). De allí que se reporten tres zonas de acuerdo a la composición florística (Centro, Norte-Sur y Río).

Tabla 5: Especies con potencial agroforestal que pueden ser incorporadas en el Departamento del Magdalena.

\begin{tabular}{|c|c|c|}
\hline Especie arbórea & Funciones en los SSP & $\begin{array}{l}\text { Referencia en Sistemas } \\
\text { agroforestales }\end{array}$ \\
\hline $\begin{array}{l}\text { Guácimo (Guazuma } \\
\text { ulmifolia) }\end{array}$ & $\begin{array}{l}\text { Reciclaje de nutrientes, follaje como alimento } \\
\text { bovino, sombrío, maderable y medicinal. }\end{array}$ & Navas (2017). \\
\hline $\begin{array}{l}\text { Campano (Albizia } \\
\text { saman). }\end{array}$ & $\begin{array}{l}\text { Alimento, sombrío, mejorador de suelos, cercas } \\
\text { vivas. }\end{array}$ & Aguirre-Morales et al., (2020). \\
\hline $\begin{array}{l}\text { Teca (Tectona } \\
\text { grandis) }\end{array}$ & $\begin{array}{l}\text { medicinal, maderable, construcción, regulación de } \\
\text { carbono atmosférico y sus hojas se han estudiado } \\
\text { como inhibidor de la corrosión. }\end{array}$ & $\begin{array}{l}\text { Tantengco et al.,(2018), } \\
\text { Manjunatha et al., (2018), } \\
\text { Kodithuwakku y Edussuriya (2018) }\end{array}$ \\
\hline $\begin{array}{l}\text { Uvito } \\
\text { (Cordia alba). }\end{array}$ & Alimento, sombra, maderable. & Navas (2017). \\
\hline $\begin{array}{l}\text { Orejero } \\
\text { (Enterolobium } \\
\text { cyclocarpum) }\end{array}$ & Maderable, sombra, fruto para ganado & Navas (2017). \\
\hline $\begin{array}{l}\text { Totumo (Crescentia } \\
\text { cujete) }\end{array}$ & $\begin{array}{l}\text { Fruto como alimento para ganado, sombra y cerca } \\
\text { viva. }\end{array}$ & Lokonon et al., (2019) \\
\hline $\begin{array}{l}\text { Aromo (Acacia } \\
\text { macracantha) }\end{array}$ & Alimento, sombra, cercas vivas, Protector de suelos. & Espejo-Díaz y Nouel-Borges (2020) \\
\hline $\begin{array}{l}\text { Trébol (Platymiscium } \\
\text { pinnatum) }\end{array}$ & $\begin{array}{l}\text { Conservación de fauna, maderable, ornamental, } \\
\text { sombrío. }\end{array}$ & Bocanegra-González et al., (2019) \\
\hline $\begin{array}{l}\text { Algarrobo } \\
\text { (Hymenaea } \\
\text { courbaril) }\end{array}$ & $\begin{array}{l}\text { Sombrío, maderable, Alimento, conservación de } \\
\text { suelos y fauna }\end{array}$ & Roca Cedeño et al., (2016). \\
\hline $\begin{array}{l}\text { Carbonero } \\
\text { (Chloroleucon } \\
\text { mangense) }\end{array}$ & Sombrío, maderable, alimento, ornamental & Romero-Duque et al., (2007) \\
\hline $\begin{array}{l}\text { Matarratón } \\
\text { (Gliricidia sepium) }\end{array}$ & Forraje, cerca viva, medicinal, protectora de suelos & Ravindran et al., (2017). \\
\hline $\begin{array}{l}\text { Ceiba (Pachira } \\
\text { quinata) }\end{array}$ & $\begin{array}{l}\text { madera, medicinal, leña, construcciones y fuente de } \\
\text { alimento para murciélagos (polinizadores) e insectos } \\
\text { esfíngidos }\end{array}$ & Werden et al., (2020) \\
\hline $\begin{array}{l}\text { Trupillo (Prosopis } \\
\text { juliflora) }\end{array}$ & Madera, forraje, cercas vivas & Shiferaw et al., (2019). \\
\hline
\end{tabular}




\section{CONCLUSIONES}

A partir de los resultados mostrados, de su análisis y de su discusión, se pueden extraer las siguientes conclusiones: 1) la estructura de especies en las 63 unidades productivas de las cuatro zonas del departamento del Magdalena es heterogénea, con diferencias en la composición florística, representada por 18 familias donde las más abundantes fueron Poaceae, Fabaceae, Bignoniaceae y Meliaceae; 2) los árboles encontrados son pocos, pero fueron priorizados 13 como potenciales para ser incorporados a sistemas agroforestales, ya que representan una opción multipropósito importante en el trópico seco donde su utilización puede proporcionar biodiversidad, belleza, reciclaje de nutrientes, sombra, alimento para el ganado y permitirán la conservación y generación de servicios ambientales como secuestro de carbono y protección de cuencas hidrográficas entre otras, y 3 ) las especies identificadas presentan características deseables para ser incluidas en sistemas agroforestales en el Departamento del Magdalena.

\section{AGRADECIMIENTOS}

Artículo derivado del proyecto "Investigación de los efectos de la variabilidad climática y el cambio climático en el recurso hídrico, biodiversidad y actividades agropecuarias en el departamento del Magdalena" Diciembre 2017 -2021. Fondo Nacional de Regalías- Gobernación del Magdalena-Universidad del Magdalena. Gracias a los productores, al equipo de profesionales y consultores del objetivo 2 por su aporte y a directivos por el financiamiento de la investigación.

\section{REFERENCIAS}

Aguirre-Morales, C. A., Thomas, E., y otros siete autores, Genetic diversity of the rain tree (Albizia saman) in Colombian seasonally dry tropical forest for informing conservation and restoration interventions, https://doi.org/10.1002/ece3.6005, Ecol. Evol., 10(4), 1905-1916 (2020)

Aguirre, S. E., Piraneque, N. V., y Díaz, C. J., Valoración del estado del suelo en zona de bosque seco tropical mediante técnicas analíticas y cromatogramas, http://dx.doi.org/10.4067/S0718-07642019000600337, Inf. Tecnol., 30(6), 337-350 (2019)

Bocanegra-González, K. T., Thomas, E., y otros cinco autores, Diversidad y estructura genética de cuatro especies arbóreas clave del bosque seco tropical en Colombia, https://doi.org/10.15446/caldasia.v41n1.71327, Caldasia, 41(1), 78-91 (2019)

Buitrago-Guillen, M. E., Ospina-Daza, L. A., y Narváez-Solarte, W., Sistemas silvopastoriles: alternativa en la mitigación y adaptación de la producción bovina al cambio climático, http://doi.org/10.17151/bccm.2018.22.1.2, Bol. Cient. Mus. Hist. Nat. U. de Caldas, 22 (1), 31-42 (2018)

Cardona, E. M., Ríos, L. A., y Peña, J. D., Disponibilidad de variedades de pastos y forrajes como potenciales materiales lignocelulósicos para la producción de bioetanol en Colombia, https://doi.org/10.4067/S0718-07642012000600010, Inf. Tecnol., 23(6), 87-96 (2012)

Espejo-Díaz, M. A., y Nouel-Borges, G. E., Evaluation of Acacia macracantha pods in balanced ration for growing rabbits, https://doi.org/10.15517/rac.v44i1.40005, Agron. Costarricense, 44(1), 93-104 (2020)

Díaz-Céspedes, M. A., Hernández-Guevara, J. E., y Gómez-Bravo, C. A., Impacto del régimen pluvial en la composición química, digestibilidad y producción de metano de Echinochloa polystachya (Kunth) Hitch, http://dx.doi.org/10.17268/sci.agropecu.2020.02.01, Scientia Agropecuaria, 11(2), 147-155 (2020)

Iglesias Gómez, J. M., Galloso-Hernández, M. A., Toral-Pérez, O. C. y Aguilar-Hernández, A., Comportamiento productivo y conducta de búfalos de río y toros Cebú en silvopastoreo, Pastos y Forrajes, 42(3), 223-229 (2019)

Khan, N., George, D., Shabbir, A., y Adkins, S., Suppresive plants as weed management tool: managing Parthenium hysterophorus under simulated grazing in Australian grasslands, https://doi.org/10.1016/j.jenvman.2019.06.051, J. of Environ. Manag., 247, 224-233 (2019)

Kodithuwakku, U., y Edussuriya, M., Jackfruit (Artocapus heterophyllus Lam.) and Teak (Tectona grandis L.) leaf extracts as green corrosion inhibitors, http://doi.org/10.4038/rjs.v9i1.35, Ruhuna J. Sci., 9, 64-69 (2018)

Lokonon, B. E. Tchandao, M., y otros cinco autores, Knowledge, valuation and prioritization of 46 woody species for conservation in agroforestry systems along Ouémé catchment in Benin (West Africa), https://doi.org/10.1007/s10668018-0142-y, Environ. Dev. Sustain., 21, 2377-2399 (2019)

Londoño, V., y Torres, A. M., Estructura y composición vegetal de un bosque seco tropical en regeneración en Bataclán (Cali, Colombia), http://dx.doi.org/10.14483/udistrital.jour.colomb.for.2015.1.a04, Colombia Forestal, 18(1), 71-85 (2015)

Mancera, K. F., Zarza H., y otros cuatro autores, Integrating links between tree coverage and cattle welfare in silvopastoral systems evaluation, https://doi.org/10.1007/s13593-018-0497-3, Agron. Sustain. Dev., 38(19), (2018)

Manjunatha M., Niveditha, M., Santhoshkumar, A., y Kunhamu, T., Simulation of Carbon Dynamics of Tectona grandis forest in Western Ghats of Kerala, India, Using Century Model, in Climate change: challenges and solutions, 247-255, Allied Publishers, Nueva Delhi, India (2018) 
Mazo, N. de los Ángeles, Rubiano, J. E., y Castro, A., Sistemas agroforestales como estrategia para el manejo de ecosistemas de bosque seco tropical en el suroccidente colombiano utilizando los SIG,

http://dx.doi.org/10.15446/rcdg.v25n1.41993, Cuadernos de Geografía, Revista Colombiana de Geografía, 25 (1), 65-77 (2016)

Molina, I. C., Angarita, E. A., y otros tres autores, Effect of Leucaena leucocephala on methane production of lucerna heifers fed a diet based on Cynodon plectostachyus, https://doi.org/10.1016/j.livsci.2016.01.009, Livestock Science, 185, 24-29 (2016)

Navas, A., Conocimiento local y diseño participativo de sistemas silvopastoriles como estrategia de conectividad en paisajes ganaderos, http://dx.doi.org/10.19052/mv.4255, Rev. Med. Vet., (34), 55-65 (2017)

Quintero-Pertúz, I., Carbonó-Delahoz, E., y Jarma-Orozco, A., Weeds associated with banana crops in Magdalena department, Colombia, https://doi.org/10.1590/s0100-83582020380100015, Planta Daninha, 38, e020217466 (2020)

Ramakrishnan, S., Kumar, S., y otros siete autores, Silvopastoral system for resilience of key soil health indicators in semi-arid environment, https://doi.org/10.1080/03650340.2020.1814954, Archives of Agronomy and Soil Science, 1-14 (2020)

Ravindran R., Juliet, S., y otros seis autores, Effects of ethanolic extract of the leaves of Pongamia glabra and Gliricidia sepium against Rhipicephalus (Boophilus) annulatus, https://dx.doi.org/10.14737/journal.aavs/2017/5.1.1.6, Adv. Anim. Vet. Sci., 5(1), 1-6 (2017)

Revueltas, J. E., Zabaleta, A., Mercado, T., y Aguirre, S., Cambios en el clima local y su efecto en la regulación hídrica en microcuencas del departamento del Magdalena, norte de Colombia, https://dx.doi.org/10.4067/S071807642020000600193, Inf. Tecnol., 31(6), 193-206 (2020)

Ríos, N., Cárdenas, A., y otros siete autores, Escorrentía superficial e infiltración en sistemas ganaderos convencionales y silvopastoriles en el trópico subhúmedo de Nicaragua y Costa Rica, Agroforestería en las Américas, 45(1), 66-71 (2006)

Roca Cedeño, A. J., Lascano, P. J., y otros ocho autores, Influencia del Algarrobo en la conducta y producción de leche de vacas en pastoreo. I. período de seca, Rev. Prod. Anim., 28(1), 1-9 (2016)

Romero-Duque, L. P., Jaramillo, V. J., y Pérez-Jiménez, A., Structure and diversity of secondary tropical dry forests in Mexico, differing in their prior land-use history, https://doi.org/10.1016/j.foreco.2007.07.002, Forest Ecology and Management, 253(1-3), 38-47 (2007)

Shiferaw, H., Bewket, W., y otros seis autores, Implications of land use/land cover dynamics and Prosopis invasion on ecosystem service values in Afar Region Ethiopia, https://doi.org/10.1016/j.scitotenv.2019.04.220, Science of the Total Environment, 675, 354-366 (2019)

Tantengco O., Condes, M., y otros tres autores, Ethnobotanical survey of medicinal plants used by Ayta communities in Dinalupihan, Bataan, Philippines, https://doi.org/10.5530/pj.2018.5.145, Pharmacognosy Journal, 10 (5), $859-870$ (2018)

Velándia, M., Boton, J., y otros siete autores, Estudio general de suelos y zonificación de tierras, departamento del Magdalena, 1a edición, 498p, Instituto Geográfico Agustín Codazzi IGAC, Bogotá, Colombia (2009)

Villanueva-López, G., Lara-Pérez, L. A., y otros cinco autores, Diversity of soil macro-arthropods correlates to the richness of plant species in traditional agroforestry systems in the humid tropics of Mexico, https://doi.org/10.1016/j.agee.2019.106658, Agriculture, Ecosystems \& Environment, 286(1), 106658 (2019)

Werden, L. K., Calderón-Morales, E., y otros cuatro autores, Using large-scale tropical dry forest restoration to test successional theory, https://doi.org/10.1002/eap.2116, Ecological Applications, 30(6), 1-17 (2020)

Zabala, W., Merino, E., y Peláez, P., Influencia de tres sistemas agroforestales del cultivo de cacao en la captura y almacenamiento de carbono, http://dx.doi.org/10.17268/sci.agropecu.2018.04.04, Scientia Agropecuaria, 9(4), 493-501 (2018) 
